\title{
Journal of Neurotrauma
}

Journal of Neurotrauma: http://mc.manuscriptcentral.com/neurotrauma

\section{Cerebrovascular signal complexity six hours after ICU admission correlates with outcome following severe traumatic brain injury}

\begin{tabular}{|r|l|}
\hline Journal: & Journal of Neurotrauma \\
\hline Manuscript ID: & NEU-2015-4228 \\
\hline Manuscript Type: & Regular Manuscript \\
\hline Date Submitted by the Author: & $19-$ Aug-2015 \\
\hline Keywords: & $\begin{array}{l}\text { Gao, Lei; Massachusetts General Hospital, Department of Anesthesia, } \\
\text { Critical Care and Pain Medicine; Cambridge University, Neurocritical Care } \\
\text { Smieleweski, Peter; Cambridge University, Neurosurgery } \\
\text { Czosnyka, Marek; Cambridge University, Neurosurgery } \\
\text { Ercole, Ari; Cambridge University, Neurocritical Care }\end{array}$ \\
\hline & \\
\hline
\end{tabular}

\section{SCHOLARONE}

Manuscripts 


\section{Full title: Cerebrovascular signal complexity six hours after ICU admission} correlates with outcome following severe traumatic brain injury

Running title: Early cerebrovascular complexity and TBI outcome

Table of Contents title: Early cerebrovascular complexity correlates with outcome following severe $\mathrm{TBI}$

Lei Gao, MD: Corresponding author

Resident Physician

Department of Anesthesia, Critical Care and Pain Medicine

Massachusetts General Hospital

55 Fruit Street, Boston, MA, 02114, USA

Igao4@partners.org

P: +1 617-888-2941, F: +1 617-726-9697

Peter Smielewski, PhD

Senior Research Associate

Division of Neurosurgery

University of Cambridge

Hills Road, Cambridge CB2 0QQ, UK

ps10011@cam.ac.uk

P: +44 1223 336946, F: +44 7869652155

Marek Czosnyka, PhD

Professor of Brain Physics

Division of Neurosurgery

University of Cambridge

Hills Road, Cambridge CB2 0QQ, UK

mc141@medschl.cam.ac.uk

P: +44 1223 336946, F: +44 7869652155

Ari Ercole, MD PhD

Consultant Neurosciences Critical Care Unit

Department of Anesthesia

University of Cambridge

Hills Road, Cambridge CB2 0QQ, UK

ae105@cam.ac.uk

P: +44 1223 217889, F: +44 7869652155 


\begin{abstract}
Disease states are associated with a breakdown in healthy interactions and are often characterised by reduced signal complexity. We applied approximate entropy (ApEn) analysis to investigate the correlation between the complexity of heart rate (ApEn-HR), mean arterial pressure (ApEn-MAP), intracranial pressure (ApEn-ICP) and a combined ApEn-Product (product of the three individual ApEns) and outcome after traumatic brain injury. In 174 severe traumatic brain injured patients we found significant differences across groups classified by the Glasgow
\end{abstract} Outcome Score in ApEn-HR $(p=0.007)$, ApEn-MAP $(p=0.02)$, ApEn-ICP $(p=0.01)$, ApEn-Product $(p=0.001)$ and $\operatorname{PRx}(p=0.004)$ in the first 6-hours. This relationship strengthened in a 24-hour and 72-hour analysis (ApEn-MAP continued to correlate with death but was not correlated with favourable outcome). Outcome was dichotomized as survival vs death, and favourable vs unfavourable; the ApEn-Product achieved the strongest statistical significance at 6-hours $(F=$ 11.0; $p=0.001$ and $F=10.5 ; p=0.001$, respectively) and was a significant independent predictor of mortality and favourable outcome $(p<0.001)$. Patients in the lowest quartile for ApEnProduct were over four times more likely to die (39.5\% vs $9.3 \%, p<0.001)$ compared to those with the highest quartile. ApEn-ICP was inversely correlated with PRx $(r=-0.39, p<0.000001)$ indicating unique information related to impaired cerebral autoregulation. Our results demonstrate that as early as 6-hours into monitoring, complexity measures from easily attainable vital signs, such as heart rate and mean arterial pressure, in addition to intracranial pressure can help triage those who require more intensive neurological management at an early stage.

Key words: complexity, intracranial pressure, approximate entropy, traumatic brain injury, autoregulation 


\title{
INTRODUCTION
}

Outcome following traumatic brain injury (TBI) has been recognised to be dependent on the initial characteristics of injury, including initial Glasgow Coma Score (GCS), pupillary reactivity, patient age, or radiological appearances; many of these characteristics are largely nonmodifiable. ${ }^{1}$ In contrast, on-going secondary brain insults remain, and are seen as potentially modifiable, and hence may affect outcome. Recent data casts doubt on ICP monitoring being superior to care based on imaging and clinical examination alone. ${ }^{2}$ Direct measurement of ICP is also not entirely without risks, and requires a surgical insertion of a sensor into the patient's brain. However when analysed continuously intracranial pressure (ICP) and ICP-derived cerebrovascular pressure reactivity are still seen as important markers of secondary insults, and their monitoring has become an established component of clinical care after traumatic brain injury. $^{3,4}$

For this reason, the search for multi-modal monitoring in TBI continues to be wide-ranging. Work involving cerebral autoregulation, ${ }^{5}$ oxygenation, ${ }^{6}$ and extracellular biochemistry ${ }^{7}$ have all been linked to outcome. Focus has been shifting towards the ICP waveform itself, which is thought to carry more information than can be described by the mean ICP alone ${ }^{8}$ One such characteristic of the ICP waveform has been examining its signal complexity.

\begin{abstract}
Higher biological organisms are recognized as complex systems composed of a network of dynamic components, each with their own regulatory mechanisms that are likely to interact with convoluted interdependence in a non-linear, non-stationary fashion. It is thought that these interactions give rise to properties that cannot wholly be understood by examining the
\end{abstract}


constituent components individually. ${ }^{9,10}$ Characterising the complexity or unpredictability of biological systems has garnered increasing interest in the medical field, and in particular, critical care. Many have observed a loss of complexity in disease states, as well as in normal ageing. ${ }^{11,12}$ For example, lower complexity of heart rate variability has been shown to be associated with poor outcome in paediatric and adult critical care patients. ${ }^{13,14}$ It has thus been postulated that complexity confers an ability to react to physiological stress and returning a system back to a fluctuating within acceptable limits; ${ }^{15}$ this may be compromised in ill health. In this study, TBI can be considered an extreme physiological stress that remains a major cause of morbidity and devastating long-term disability in a relatively young population.

Since its introduction by Pincus, Approximate entropy (ApEn) has become a widely used as a non-linear, entropy formula for estimating the amount of complexity (irregularity and unpredictability of fluctuations) in time-series data. ${ }^{16}$ It determines the conditional probability of similarity between a chosen data segment of a given duration, and the next set of segments of the same duration; the higher the probability of similarity, the smaller the ApEn value, indicating lower complexity. ${ }^{17} \mathrm{ApEn}$ of biological time signals has been successfully used to distinguish healthy from pathological states such as ventricular dysfunction, respiratory failure, endocrine disorders, and sleep apnoea. In neuropathology, analysis of the electroencephalogram using ApEn has helped characterise patients with Alzheimer's, ${ }^{18}$ epilepsy, ${ }^{19}$ and schizophrenia. ${ }^{20}$

There is evidence that decreased intracranial pressure complexity coincides with intracranial hypertension in children ${ }^{21}$ and adults ${ }^{22}$ after head injury. Low complexity in the ICP waveform has been shown to be a significant predictor of mortality and poor outcome after TBI. ${ }^{23}$ Others have used heart rate and blood pressure variability to predict poor outcome in general trauma ${ }^{24}$ ${ }^{25}$ and predict mortality in a small cohort of traumatic brain injury patients. ${ }^{26}$ To date, no study 
has examined complexity using ApEn simultaneously for both cerebral (ICP) and cardiovascular (MAP and HR) signals in a large cohort of TBI patients, especially early post-ictus. Our aim was to explore whether early complexity measures correlated with neurological outcome within the first 6-hour, 24-hour, and 72-hour periods of monitoring in the intensive care unit (ICU). We hypothesize that whilst HR, MAP and ICP are linked, a combined measure of complexity ApEnProduct contains more information related to outcome, whilst individually, ApEn-ICP may still yield distinct information about the intracranial injury not captured by the systemic counterparts.

\section{MATERIAL AND METHOD}

\section{Data acquisition}

Anonymised data recordings made as part of routine care from patients admitted to the neurosciences ICU at Addenbrookes Hospital in Cambridge after severe head injury between 2002 and 2011 were selected for retrospective analysis. Informed consent was obtained from all patients (or their next of kin) for the use of collected data for research purposes. The study was approved by the relevant research ethics committee (29 REC 97/291). Included patients had at least 72 hours of ICP, MAP and HR recording started within 24 hours of admission, along with documented GCS, and Glasgow Outcome Scale (GOS) at six months. Those with over one hour of missing recordings (prolonged scan times, technical issues during recording, re-operation etc.) were excluded from analysis. All patients were sedated, mechanically ventilated and managed according to a cerebral perfusion pressure (CPP) orientated protocol ${ }^{27}$ during their stay on ICU. 
Data was acquired through standard monitoring kit for ICP (intraparenchymal probe, Codman \& Shurtleff Inc., MA, USA), MAP (mainly radial artery, Baxter Healthcare Corp, CA, USA), and HR (via routine cardiac monitoring. All signals were continuously sampled using ICM+ software (Cambridge, UK, http://www.neurosurg.cam.ac.uk/icmplus) at a frequency between 30 to 200 $\mathrm{Hz}$. In order to suppress pulse and respiratory waves and focus entirely on the slow fluctuations of ICP consecutive 10-second averages were calculated. Changes in ICP and MAP over 30 consecutive 10s time averages were used to calculate the pressure reactivity index (PRx), a moving Pearson correlation coefficient.

\section{Approximate entropy analysis}

ApEn was calculated using freely available Matlab script based on a method first described by Pincus. ${ }^{16}$ ApEn requires two input parameters: segment length $m$, and a tolerance $r$ (set to a certain percentage of the standard deviation (SD) of the time-series). In brief, ApEn analysis determines the conditional probability of similarity between a defined data segment of a given duration, and the next set of segments of the same duration within a pre-defined tolerance window; the higher the probability, the smaller the ApEn value, indicating less complexity of the data ${ }^{17}$ (see illustrative example in Figure 1). The most widely used values have been $m=1$ or $m$ $=2$, and $r=20 \%,{ }^{28}$ whereas other studies have successfully used wider values for $\mathrm{m}$ and $r .^{29}$ We used $m=2$ and $r=20 \%$ as selecting these values maximised the ApEn based on our preliminary data analysis. After 10-second averaging, each 1-hour period produced 360 data points for analysis. Calculations were performed on the first 6-hour, 24-hour, and 72-hour periods for each of the 174 patients. 


\section{Statistical analysis}

The PRx, mean values and ApEn of ICP, MAP, and HR for all 174 patients over the three $24 \mathrm{~h}$ periods were included for analysis using IBM's SPSS 20. An ApEn Product was also calculated from the product of the 3 individual ApEn values for each patient. Outcome was assessed at 6 months after head injury using Glasgow Outcome Scale; ${ }^{30}$ due to low number of subjects $(n=5)$, those with persistent vegetative state were analysed with the severe disablitily group). Groups were further dichotomised into survival versus death, as well as favourable versus unfavourable outcome (good recovery or moderate disability versus severe disability or death). Interval data are expressed as mean \pm SD or $95 \%$ confidence interval, or median with interquartile range, and compared with one-way ANOVA, or Kruskal-Wallis non-parametric test as appropriate. Categorical data were compared using chi-squared testing. One-way ANOVA was used to compare interval variables between the dichotomised groups. Pearson's correlation was used to examine correlation between continuous variables. A multivariable logistic regression model was used to identify independent predictors for the dichotomised outcomes. Variables were normalised as appropritate if found not to follow a normal distribution before inclusion in the logistic regression model. $P<0.05$ was chosen to represent a significant difference. 


\section{RESULTS}

\section{Patient demographics}

Of 174 patients included in the study, $131(75 \%)$ of patients were male and $43(25 \%)$ were

female (Table 1). The average age was $37.8 \pm 15$. Based on the initial post-resuscitation Glasgow Coma Scale, 110 (63\%) sustained severe (GCS $\leq 8$ ) head injury. At six months post injury, 132 (76\%) survived and 42 died.

\section{Correlation of complexity with outcome}

Figure 2 summarizes the studied parameters for different outcome groups during the first 6 hour analysis period. In a cohort analysis, there was no statistical difference for intracranial pressure (ICP) or cerebral perfusion pressure (CPP) across outcome groups during any time period. The pressure reactivity index (PRx) was significantly higher in the fatal group during all time periods ( $p<0.01, p<0.001$ and $p<0.01$, respectively). When the ApEn was calculated for ICP (ApEn-ICP), mean arterial pressure (ApEn-MAP) and heart rate (ApEn-HR), all three parameters were significantly lower in the fatal group in the first 6 hours (Figure 2; $p<0.01, p<$ 0.05 , and $p<0.01$, respectively). The fatal group continued to have significantly lower ApEn-ICP $(p<0.001)$ and ApEn-HR $(p<0.01)$ while ApEn-MAP was not in the 24 -hour analysis $(p>0.05)$. For the 72-hour analysis, only ApEn-ICP was significantly lower in the fatal group $(p<0.0001)$.

Table 2 shows the differences in our study parameters when patients are dichotomised into survival vs. death and favourable vs. unfavourable using one-way ANOVA. A higher F-value suggests stronger differentiation of a parameter across different outcome groups. All ApEn 
parameters had significant $F$-values for 6-hour, 24-hour, and 72-hour analysis time periods irrespective of dichotomisation of outcome groups; the exception was for ApEn-MAP for favourable vs. unfavourable during the 24 -hour period $(F=3.8 ; P=0.05)$ and the 72 -hour period $(F=2.9 ; P=0.09)$. The ApEn-Product (ApEn-ICP $\times$ ApEn-MAP $\times$ ApEn-HR) was calculated to incorporate the three complexity measures. We found it to have the highest $F$-value during the 6-hour period for survival vs. death $(F=11.0 ; P=0.001)$ and favourable vs. unfavourable $(F=$ $10.5 ; P=0.001)$. This relationship was seen again in the 24 -hour period, and only ApEn-ICP more strongly differentiated survival vs. death during the 72 -hour period $(F=16.4 ; P=0.00008$ versus $F=15.9 ; P=0.0001)$

\section{Complexity as an independent predictor of outcome}

We found age $(p<0.01)$, presenting Glasgow Coma Scale $(p<0.05)$, and the pressure reactivity index $(p<0.05)$ to be significantly associated with mortality using a multivariable logistic regression (Table 3) when approximate entropy parameters are excluded from analysis. All approximate entropy parameters were then found to be significant predictors of mortality at 6hour (Table 3A), 24-hour, and 72-hour periods. However, introducing more than one ApEn parameter rendered all ApEn parameters insignificant suggesting the presence of co-linearity.

When ApEn parameters during 6-hour analysis were excluded from the model to predict mortality, the accuracy, sensitivity and specificity were $0.78 \pm 0.05,0.19 \pm 0.12$ and $0.97 \pm 0.07$ respectively. When ApEn-Product during 6-hour analysis was included, the accuracy, sensitivity and specificity were $0.81 \pm 0.06,0.29 \pm 0.11$ and $0.97 \pm 0.07$ respectively. The sensitivity was higher with ApEn-Product measured in the 24-hour $(0.33 \pm 0.12)$ and 72 -hour analysis $(0.31 \pm$ 0.12 ) but these differences were within errors. The ApEn-Product was the strongest predictor 
throughout. PRx became insignificant throughout when ApEn-ICP or ApEn Product was introduced. Similarly, in multivariable logistic regression for prediction of favourable outcome, all approximate entropy parameters were significant predictors in all analysis periods, except for ApEn-MAP in the 72-hour period. Shown in Table 3B are factors predictive of favourable outcome in the first 6-hour period. PRx was not a significant independent predictor of favourable outcome in all three analyses $(p>0.05)$. Mean ICP and CPP were also not found to be significant predictors during any analysis period.

Correlation between complexity and the pressure reactivity index

We found the ApEn parameters to be significantly correlated to each other in all analysis periods, with ApEn-MAP and ApEn-HR being the strongest ( $r=0.62, p<0.000001$ in the 24-hour analysis). This suggests a degree of overlap in information regarding the state of the biological system. The ApEn-ICP was inversely correlated with PRx (Figure 3A; $r=-0.39, p<0.000001$ ) indicating lower complexity during impaired cerebral autoregulation (high, positive PRx). No correlation was seen between ApEn-MAP or ApEn-HR and PRx (Figure 3B and 3C, respectively).

\section{DISCUSSION}

A prior study showed that higher mean intracranial pressure, intact cerebral autoregulation as inferred from a lower, more negative pressure reactivity index (PRx) ${ }^{31}$ and lower intracranial pressure (ICP) complexity over the whole intensive care monitoring period was associated with poor outcome. ${ }^{23}$ In this study, we show that the mean arterial pressure (MAP) and heart rate (HR) complexity in addition to the ICP complexity was highest in patients with good outcome, 
and lowest in patients with fatal outcomes. This finding supports the notion that complexity is a broader reflection on the global health of the physiological system. ${ }^{32}$ We also established the relationship between complexity and outcome in traumatic brain injury exists as early as the first 6-hours of monitoring.

We know that outcome from head injury is thought to be dependent on two broad mechanisms: (a) the primary insult, both cranial and extra-cranial damage occurring at the moment of impact, and (b) the secondary insult where pathological processes initiated at the moment of injury manifest with delayed clinical presentation over the first few days. These secondary insults include imbalance between CBF and metabolism, excitotoxicity, oedema formation, and inflammatory and apoptotic processes. ${ }^{33}$ We postulate that the lower ApEn-MAP and ApEn-HR in those who do worse may be a reflection on the severity of the initial extra-cranial insult, whereas the lower ApEn-ICP is more of a reflection on the severity of the primary cranial insult. Other studies have shown that heart rate complexity and blood pressure variability predicts poor outcome in general trauma. ${ }^{24}{ }^{25}$ In our study, ApEn-MAP and ApEn-HR had lower predictive value in the 72-hour period, perhaps a sign of cardiovascular resuscitation and stabilisation of the extra-cranial insult, although there is no way to exclude the effects of patients weaning from the ventilator.

We showed that ApEn-ICP is weakly correlated with ApEn-MAP, while ApEn-MAP and ApEn-HR are more strongly correlated. Only ApEn-ICP is significantly correlated with PRx. This supports our inference that the complexity of MAP and HR perhaps reflects 'cardiovascular' health, whereas the complexity of ICP is more closely related to 'cerebral' health and intact autoregulatory mechanisms. However, the basis of current TBI management links these two 
systems in the fundamental cerebral perfusion pressure equation; it may not be a surprise that while there is a degree of overlap, there must also exist unique prognostic information, perhaps explaining why our ApEn-Product, simply the product of the 3 ApEn parameters, more strongly separates survival/death and favourable/unfavourable groups.

Our results demonstrate that patients with lower ApEn after 6-hours of monitoring were more likely to do poorly. To put this into perspective in Figure 4, patients with the lowest quartile for ApEn-Product experienced a $39.5 \%$ (17/43) chance of dying and only $27.9 \%$ (12/43) chance of favourable outcome compared with $9.3 \%(4 / 43 ; p<0.005)$ and $60.5 \%(26 / 43 ; p<0.01)$ in the lowest quartile, respectively. ApEn-ICP followed by ApEn-HR were the strongest performers of the individual signal ApEns. This discriminating power for PRx in mortality was similar to ApEnICP and weaker than ApEn-Product. However, it was not an independent predictor of favourable outcome in any time period in our analysis even though it has been shown to be when the whole ICU monitoring period was analysed. ${ }^{23}$ This is surprising given that 72 -hours should be sufficient for impaired autoregulation to manifest and makes ApEn (especially ApEn-ICP) a useful early adjunct to PRx. Clinically, these results can help triage those who require more intensive neurological management at an earlier stage. The fact that complexity of heart rate and mean arterial pressure are related to neurological outcome suggests they can potentially be used as early triage of patients who are likely to do poorly especially early on where ICP monitoring may not yet be available e.g. prior to transport, or even informing decision to transfer a patient to specialist neurosurgical care.

Within our 72-hour period, neither mean ICP nor CPP were associated with outcome. There has been on going research over the role of ICP monitoring in TBI. ${ }^{2} \mathrm{~A}$ detailed debate is not our 
intention in this study, but our results do suggest that while ICP can be seen as a gross measure of end-organ injury, and being exposed to a higher value over the whole ICU stay clearly does predict poor outcome, ${ }^{23}$ there is not enough information to predict outcome in the first 72 hours. Rather, high ICP is likely to occur later in those that do poorly and may reflect patients who have difficult to control values. This highlights the potential additional value of early measurements of complexity not only of ICP, but also cardiovascular complexity via MAP and HR monitoring that reveal crucial prognostic information beyond the original absolute values.

Using a multivariable logistic regression model we have shown that complexity of ICP, MAP, and HR are predictors of mortality and favourable outcome independent of age, initial GCS, mean ICP, CPP and PRx (with either ApEn-MAP and ApEn-HR included in our model) early in the course of TBI. We propose that incorporating multiple complexity signals may provide better insight into the 'health' of a biological system. Bashan et al argue that the human organism is an integrated network where complex physiological systems, each with its own regulatory mechanisms, continuously interact, and where failure of one system can trigger a breakdown of the entire network. ${ }^{10}$ This is perhaps not surprising given that the ICP, MAP, and HR signals themselves are likely a reflection of thousands of individual processes that are interlinked in a complex network, with some mutually dependent, whilst others are independent. There may be unique information about the health of a biological system when the network as a whole is considered, and merits further study in the setting of TBI. 


\section{LIMITATIONS}

This is a retrospective, observational study where it was not feasible to control for the effects of clinical interventions (e.g. CSF drainage or medications, weaning from the vent) on complexity. Although all patients were monitored on a single neuro-critical care unit where therapeutic interventions were standardized, ${ }^{27}$ variation inevitably occurs. To minimise the variability of when patients arrived in the ICU relative to time of injury, we included only those patients arriving within 24 hours of head injury.

\section{CONCLUSION}

We showed that there is a significant relationship between the complexity of intracranial pressure, mean arterial pressure and heart rate with outcome after severe traumatic brain injury, in the first 6-hour monitoring period where those more likely to do poorly can be identified early; this relationship continues in the first 24 -hour and first 72 -hour period. We identify complexity as an independent predictor of mortality and favourable outcome. There is significant colinearity among the complexity parameters. However our work suggests that ApEnICP yields some information that is distinct from the MAP and HR counterparts and an inverse relationship between the ApEn-ICP and the PRx suggests that this may reflect autoregulation. Early (6-hour) outcome discrimination with ApEn-Product was the strongest. Using complexity of multiple signals is a stronger correlate with outcome suggesting further work should be directed at the changes in the network as a whole in TBI patients. 


\section{DISCLOSURES}

ICM+ software (www.neurosurg.cam.ac.uk/icmplus) is licensed by Cambridge Enterprise Ltd, UK.

Authors PS and MC have financial interest in a fraction of licensing fee. 


\section{REFERENCES}

1. Murray, G.D., Butcher, I., McHugh, G.S., Lu, J., Mushkudiani, N.A., Maas, A.I.R., Marmarou, A., and Steyerberg, E.W. (2007). Multivariable prognostic analysis in traumatic brain injury: results from the IMPACT study. J. Neurotrauma 24, 329-37.

2. Chesnut, R.M., Temkin, N., Carney, N., Dikmen, S., Rondina, C., Videtta, W., Petroni, G., Lujan, S., Pridgeon, J., Barber, J., Machamer, J., Chaddock, K., Celix, J.M., Cherner, M., and Hendrix, T. (2012). A trial of intracranial-pressure monitoring in traumatic brain injury. N. Engl. J. Med. 367, 2471-81.

3. Aries, M.J.H., Czosnyka, M., Budohoski, K.P., Steiner, L.A., Lavinio, A., Kolias, A.G., Hutchinson, P.J., Brady, K.M., Menon, D.K., Pickard, J.D., and Smielewski, P. (2012). Continuous determination of optimal cerebral perfusion pressure in traumatic brain injury. Crit. Care Med. 40, 2456-63.

4. Smith, M. (2008). Monitoring intracranial pressure in traumatic brain injury. Anesth. Analg. 106, 240-8.

5. Czosnyka, M., Smielewski, P., Kirkpatrick, P., Laing, R.J., Menon, D., and Pickard, J.D. (1997). Continuous assessment of the cerebral vasomotor reactivity in head injury. Neurosurgery 41, 11-7; discussion 17-9.

6. Van den Brink, W.A., van Santbrink, H., Steyerberg, E.W., Avezaat, C.J., Suazo, J.A., Hogesteeger, C., Jansen, W.J., Kloos, L.M., Vermeulen, J., and Maas, A.I. (2000). Brain oxygen tension in severe head injury. Neurosurgery 46, 868-76; discussion 876-8. 
7. Timofeev, I., Carpenter, K.L.H., Nortje, J., Al-Rawi, P.G., O'Connell, M.T., Czosnyka, M., Smielewski, P., Pickard, J.D., Menon, D.K., Kirkpatrick, P.J., Gupta, A.K., and Hutchinson, P.J. (2011). Cerebral extracellular chemistry and outcome following traumatic brain injury: a microdialysis study of 223 patients. Brain 134, 484-94.

8. Czosnyka, M., Smielewski, P., Timofeev, I., Lavinio, A., Guazzo, E., Hutchinson, P., and Pickard, J.D. (2007). Intracranial pressure: more than a number. Neurosurg. Focus 22, E10.

9. Gallagher, R. (1999). Beyond Reductionism. Science (80-. ). 284, 79-79.

10. Bashan, A., Bartsch, R., and Kantelhardt, J. (2012). Network physiology reveals relations between network topology and physiological function. Nat. ... 3, 702.

11. Goldberger, A.L., Peng, C.-K., and Lipsitz, L. a. (2002). What is physiologic complexity and how does it change with aging and disease? Neurobiol. Aging 23, 23-6.

12. Lipsitz, L.A., and Goldberger, A.L. (1992). Loss of "complexity" and aging. Potential applications of fractals and chaos theory to senescence. JAMA 267, 1806-9.

13. Goldstein, B., Fiser, D.H., Kelly, M.M., Mickelsen, D., Ruttimann, U., and Pollack, M.M. (1998). Decomplexification in critical illness and injury: Relationship between heart rate variability, severity of illness, and outcome. Crit. Care Med. 26.

14. Norris, P.R., Anderson, S.M., Jenkins, J.M., Williams, A.E., and Morris, J. a. (2008). Heart rate multiscale entropy at three hours predicts hospital mortality in 3,154 trauma patients. Shock 30, 17-22. 
15. Que, C.L., Kenyon, C.M., Olivenstein, R., Macklem, P.T., and Maksym, G.N. (2001). Homeokinesis and short-term variability of human airway caliber. J. Appl. Physiol. 91, $1131-41$

16. Pincus, S.M. (1991). Approximate entropy as a measure of system complexity. Proc. Natl. Acad. Sci. 88, 2297-2301.

17. Chon, K., Scully, C.G., and Lu, S. (2009). Approximate entropy for all signals. IEEE Eng. Med. Biol. Mag. 28, 18-23.

18. Abásolo, D., Hornero, R., Espino, P., Poza, J., Sánchez, C.I., and de la Rosa, R. (2005). Analysis of regularity in the EEG background activity of Alzheimer's disease patients with Approximate Entropy. Clin. Neurophysiol. 116, 1826-34.

19. Srinivasan, V., Eswaran, C., and Sriraam, N. (2007). Approximate entropy-based epileptic EEG detection using artificial neural networks. IEEE Trans. Inf. Technol. Biomed. 11, 28895.

20. Sabeti, M., Katebi, S., and Boostani, R. (2009). Entropy and complexity measures for EEG signal classification of schizophrenic and control participants. Artif. Intell. Med. 47, 26374.

21. Hornero, R., Aboy, M., Abasolo, D., McNames, J., Wakeland, W., and Goldstein, B. (2006). Complex analysis of intracranial hypertension using approximate entropy. Crit. Care Med. 34, 87-95. 
22. Hornero, R., Aboy, M., Abásolo, D., McNames, J., and Goldstein, B. (2005). Interpretation of approximate entropy: analysis of intracranial pressure approximate entropy during acute intracranial hypertension. IEEE Trans. Biomed. Eng. 52, 1671-80.

23. Lu, C.-W., Czosnyka, M., Shieh, J.-S., Smielewska, A., Pickard, J.D., and Smielewski, P. (2012). Complexity of intracranial pressure correlates with outcome after traumatic brain injury. Brain 135, 2399-408.

24. Norris, P.R., Stein, P.K., and Morris, J.A. (2008). Reduced heart rate multiscale entropy predicts death in critical illness: a study of physiologic complexity in 285 trauma patients. J. Crit. Care 23, 399-405.

25. Norris, P.R., Canter, J.A., Jenkins, J.M., Moore, J.H., Williams, A.E., and Morris, J.A. (2009). Personalized medicine: genetic variation and loss of physiologic complexity are associated with mortality in 644 trauma patients. Ann. Surg. 250, 524-30.

26. Papaioannou, V., Giannakou, M., Maglaveras, N., Sofianos, E., and Giala, M. (2008). Investigation of heart rate and blood pressure variability, baroreflex sensitivity, and approximate entropy in acute brain injury patients. J. Crit. Care 23, 380-6.

27. Menon, D.K. (1999). Cerebral protection in severe brain injury: physiological determinants of outcome and their optimisation. Br. Med. Bull. 55, 226-258.

28. Pincus, S., and Kalman, R.E. (2004). Irregularity, volatility, risk, and financial market time series. Proc. Natl. Acad. Sci. U. S. A. 101, 13709-14. 
29. Hornero, R., Alvarez, D., Abasolo, D., Gomez, C., del Campo, F., and Zamarron, C. (2005). Approximate entropy from overnight pulse oximetry for the obstructive sleep apnea syndrome. Conf. Proc. IEEE Eng. Med. Biol. Soc. 6, 6157-60.

30. Jennett, B., and Bond, M. (1975). Assessment of outcome after severe brain damage. Lancet 1, 480-4.

31. Brady, K.M., Lee, J.K., Kibler, K.K., Easley, R.B., Koehler, R.C., and Shaffner, D.H. (2008). Continuous measurement of autoregulation by spontaneous fluctuations in cerebral perfusion pressure: comparison of 3 methods. Stroke. 39, 2531-2537.

32. Buchman, T.G. (2004). Nonlinear dynamics, complex systems, and the pathobiology of critical illness. Curr. Opin. Crit. Care 10, 378-82.

33. Werner, C., and Engelhard, K. (2007). Pathophysiology of traumatic brain injury. Br. J. Anaesth. 99, 4-9. 
Table 1. Patient demographics for 174 subjects

\begin{tabular}{lccccc}
\hline & $\begin{array}{c}\text { Good } \\
\text { Outcome }\end{array}$ & $\begin{array}{c}\text { Moderate } \\
\text { Disability }\end{array}$ & $\begin{array}{c}\text { Severe } \\
\text { Disability }\end{array}$ & Death & $P$ value \\
\hline Number & 27 & 47 & 58 & 42 & \\
Age (years) & $34.4 \pm 16.6$ & $32.9 \pm 12.7$ & $38.4 \pm 13.7$ & $44.4 \pm 16.0$ & $0.0021^{*}$ \\
Male (\%) & 55.6 & 80.9 & 74.1 & 83.3 & $0.047^{*}$ \\
GCS & 8 (IQR 5-9) & 8 (IQR 4-11) & 5 (IQR 3-8) & 4 (IQR 3-8) & $0.0036^{*}$ \\
$\begin{array}{l}\text { Monitoring length } \\
\text { (days) }\end{array}$ & $6.8 \pm 3.7$ & $7.6 \pm 3.7$ & $7.3 \pm 3.2$ & $7.2 \pm 3.5$ & 0.81 \\
\hline $\begin{array}{l}\text { Good Outcome = GOS 5, Moderate Disability = 4, Severe Disability = 3 and 2, Death = 1 } \\
\text { GOS Glasgow Outcome Score, GCS Glasgow Coma Scale. }\end{array}$ & \\
$\begin{array}{l}\text { Numerical data expressed as mean } \pm \text { SD and compared with ANOVA. Categorical data expressed } \\
\text { as number (percentage) or median (IQR) and compared with chi-squared test. }\end{array}$
\end{tabular}


Table 2. Differences in measured parameters related to outcome using one-way ANOVA

A. First 6-hour

\begin{tabular}{|c|c|c|c|c|}
\hline \multirow{2}{*}{$\begin{array}{l}\text { Parameter } \\
(n=174)\end{array}$} & \multicolumn{2}{|c|}{ Survival vs Death } & \multicolumn{2}{|c|}{ Favorable vs Unfavorable } \\
\hline & $F$ & $P$ & $F$ & $P$ \\
\hline \multicolumn{5}{|l|}{ 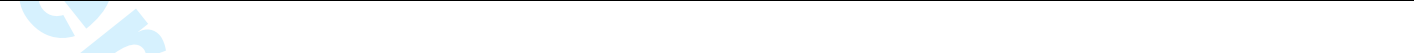 } \\
\hline ICP & 0.4 & 0.5 & 0.3 & 0.6 \\
\hline CPP & 0.3 & 0.6 & 0.5 & 0.3 \\
\hline PRx & 7.3 & $0.008 *$ & 4.7 & $0.03 *$ \\
\hline ApEn-ICP & 6.9 & $0.009 *$ & 5.1 & $0.03 *$ \\
\hline ApEn-MAP & 6.9 & $0.009 *$ & 6.6 & $0.01 *$ \\
\hline ApEn-HR & 6.5 & $0.01^{*}$ & 8.8 & $0.003^{*}$ \\
\hline ApEn-Product & 11.0 & $0.001 *$ & 10.5 & $0.001 *$ \\
\hline
\end{tabular}

B. First 24-hour

\begin{tabular}{lcccc}
\hline Parameter & \multicolumn{2}{c}{ Survival vs Death } & \multicolumn{2}{c}{ Favorable vs Unfavorable } \\
$(\mathrm{n}=174)$ & $F$ & $P$ & $F$ & $P$ \\
\hline & & & & \\
ICP & 0.07 & 0.8 & 0.2 & 0.7 \\
CPP & 0.1 & 0.74 & 0.6 & 0.32 \\
PRx & 13.8 & $\mathbf{0 . 0 0 0 3 ^ { * }}$ & 4.3 & $\mathbf{0 . 0 4 *}$ \\
ApEn-ICP & 11.6 & $\mathbf{0 . 0 0 0 8 ^ { * }}$ & 5.4 & $\mathbf{0 . 0 2}$ \\
ApEn-MAP & 4.3 & $\mathbf{0 . 0 4 ^ { * }}$ & 3.8 & 0.05 \\
ApEn-HR & 8.9 & $\mathbf{0 . 0 0 3 ^ { * }}$ & 8.2 & $\mathbf{0 . 0 0 5 ^ { * }}$ \\
ApEn-Product & 15.0 & $\mathbf{0 . 0 0 0 1 ^ { * }}$ & 13.0 & $\mathbf{0 . 0 0 0 4 ^ { * }}$ \\
& & & & \\
\hline
\end{tabular}

C. First 72-hour

\begin{tabular}{|c|c|c|c|c|}
\hline \multirow{2}{*}{$\begin{array}{l}\text { Parameter } \\
(\mathrm{n}=174)\end{array}$} & \multicolumn{2}{|c|}{ Survival vs Death } & \multicolumn{2}{|c|}{ Favorable vs Unfavorable } \\
\hline & $F$ & $P$ & $F$ & $P$ \\
\hline ICP & 1.2 & 0.3 & 0.001 & 0.9 \\
\hline CPP & 1.0 & 0.3 & 0.4 & 0.5 \\
\hline PRx & 10.2 & $0.002 *$ & 2.5 & 0.1 \\
\hline ApEn-ICP & 16.4 & $0.00008^{*}$ & 6.1 & $0.01 *$ \\
\hline ApEn-MAP & 5.9 & $0.02 *$ & 2.9 & 0.09 \\
\hline ApEn-HR & 8.6 & $0.004^{*}$ & 4.3 & $0.04^{*}$ \\
\hline ApEn-Product & 15.9 & $0.0001 *$ & 8.4 & $0.004^{*}$ \\
\hline
\end{tabular}


Table 3. Multivariate logistic regression for the first 6-hour period

A. Factors associated with mortality in patients after traumatic brain injury

\begin{tabular}{|c|c|c|c|c|c|c|}
\hline \multirow{2}{*}{8} & \multicolumn{3}{|c|}{ Without ApEn-Product } & \multicolumn{3}{|c|}{ With ApEn-Product } \\
\hline & OR & $95 \% \mathrm{Cl}$ & $P$-value & OR & $95 \% \mathrm{Cl}$ & $P$-value \\
\hline Age & 1.04 & $1.01-1.07$ & 0.003 & 1.04 & $1.01-1.07$ & 0.003 \\
\hline $\mathrm{GCS} \leq 8$ & 2.41 & $1.06-5.48$ & 0.04 & 2.37 & $1.03-5.44$ & 0.04 \\
\hline$P R x^{a}$ & 5.45 & $1.07-27.8$ & 0.04 & 4.09 & $0.78-21.5$ & 0.1 \\
\hline ApEn-Product & NA & NA & NA & 0.55 & $0.36-0.86$ & 0.008 \\
\hline
\end{tabular}

B. Factors associated with favourable outcome in patients after traumatic brain injury

\begin{tabular}{|c|c|c|c|c|c|c|}
\hline & \multicolumn{3}{|c|}{ Without ApEn-Product } & \multicolumn{3}{|c|}{ With ApEn-Product } \\
\hline & OR & $95 \% \mathrm{Cl}$ & $P$-value & OR & $95 \% \mathrm{Cl}$ & $P$-value \\
\hline Age & 0.96 & $0.94-0.98$ & 0.001 & 0.96 & $0.94-0.98$ & 0.001 \\
\hline $\mathrm{GCS} \leq 8$ & 0.23 & $0.11-0.46$ & 0.0004 & 0.21 & $0.10-0.45$ & 0.0006 \\
\hline PRx & 0.28 & $0.06-1.25$ & 0.09 & NA & NA & NA \\
\hline ApEn-Product & NA & NA & NA & 1.64 & $1.15-2.33$ & 0.007 \\
\hline
\end{tabular}

GCS = Glasgow Coma Scale; PRx = pressure reactivity index; ApEn-Product $=$ approximate entropy product; $\mathrm{NA}=$ not applicable; $\mathrm{OR}=$ odds ratio; $\mathrm{Cl}=$ confidence interval 
Figure 1

A

Data series $\mathrm{u}(\mathrm{i})$ from $\mathrm{i}=1$ to $\mathrm{N}$

Split data into runs of $\mathrm{m}=1$

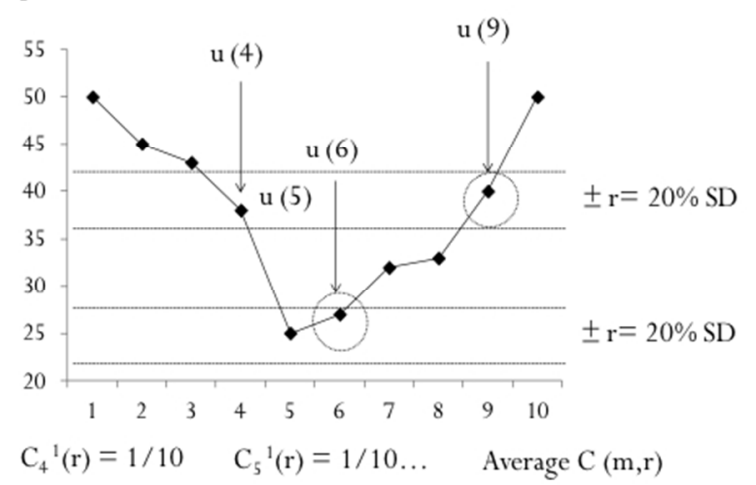

Measure of prevalence of repetitive patterns, $m$

B Split data into runs of $\mathrm{m}+1=2$

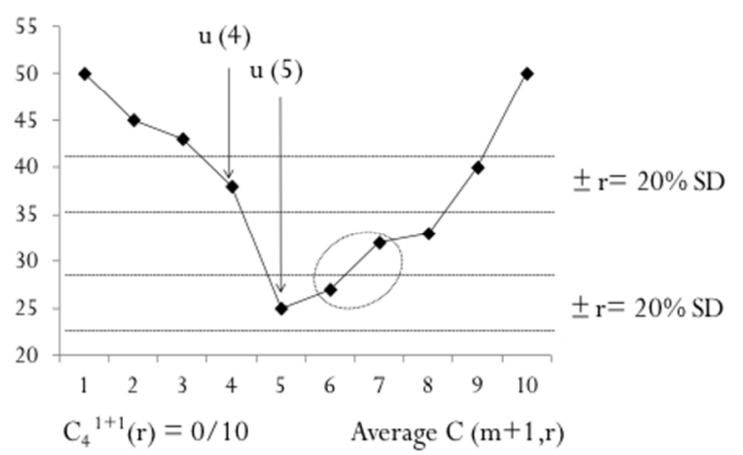

Measure of prevalence of repetitive patterns, $\mathrm{m}+1$

$254 \times 338 \mathrm{~mm}(72 \times 72$ DPI $)$ 
Figure 2
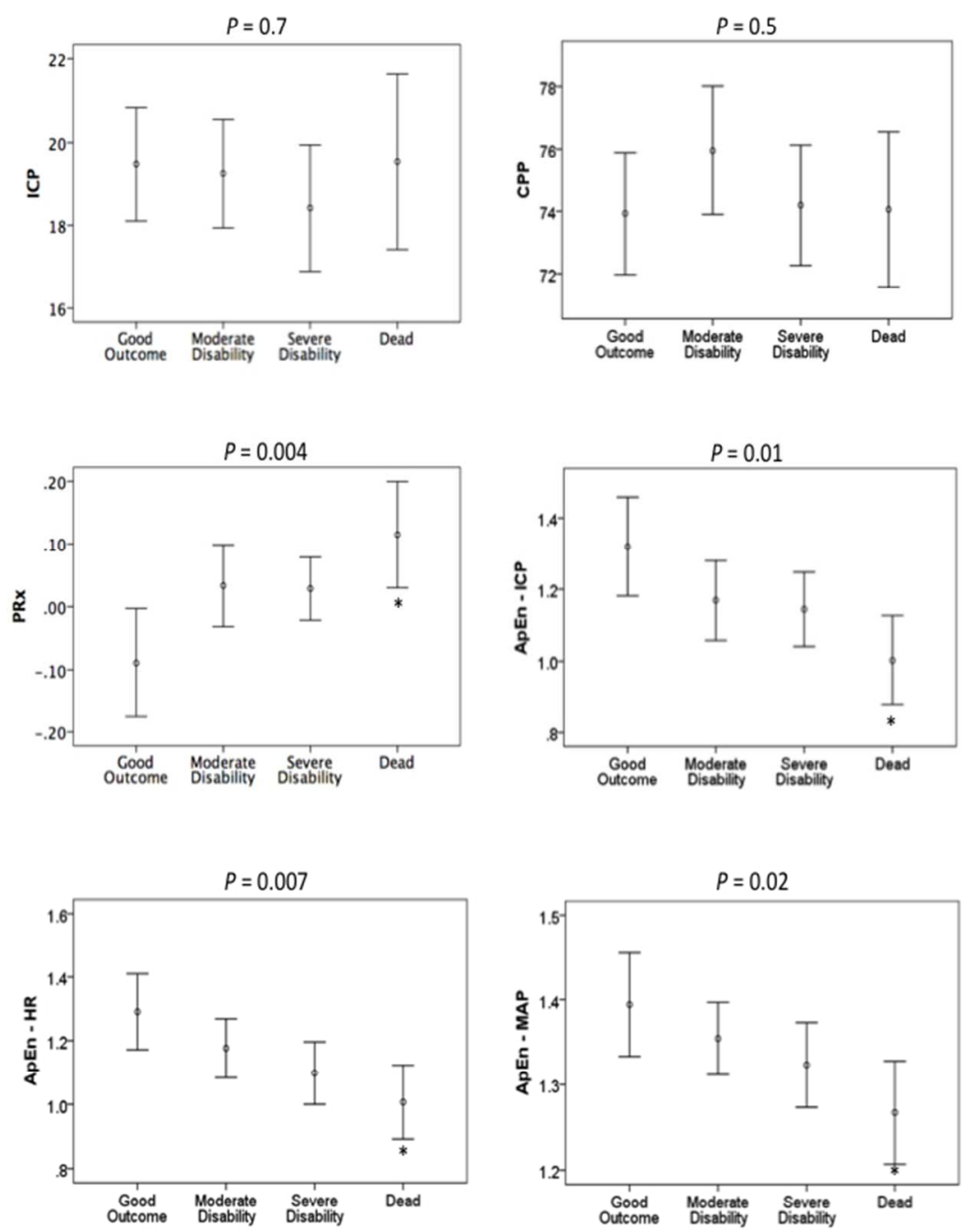

$254 \times 338 m m(72 \times 72$ DPI $)$

Mary Ann Liebert, Inc, 140 Huguenot Street, New Rochelle, NY 10801 
Figure 3

A
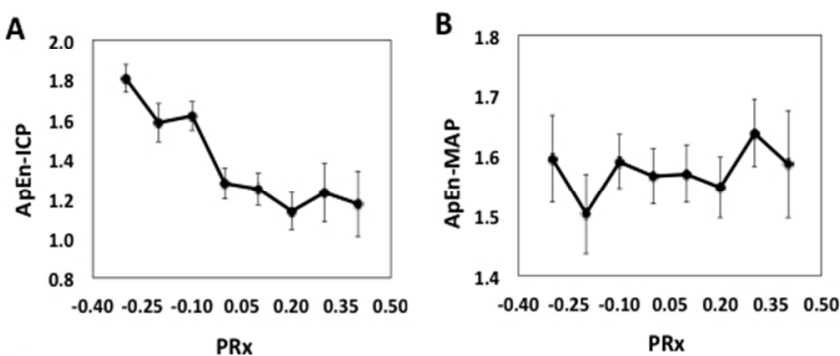

C

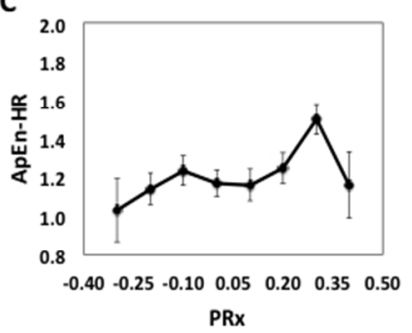

30

31

32

33

34

35

36

37

38

39

40

41

42

43

44

45

46

47

48

49

50

51

52

53

54

55

56

57

58

59

60

$254 \times 338 m m(72 \times 72$ DPI $)$ 


1
2
3
4
5
6
7
8
9
10
11
12
13
14
15
16
17
18
19
20
21
22
23
24
25
26
27
28
29
30
31
32
33
34
35
36
37
38
39
40
41
42
43
44
45
46
47
48
49
50
51
52
53
55
50

Figure 4

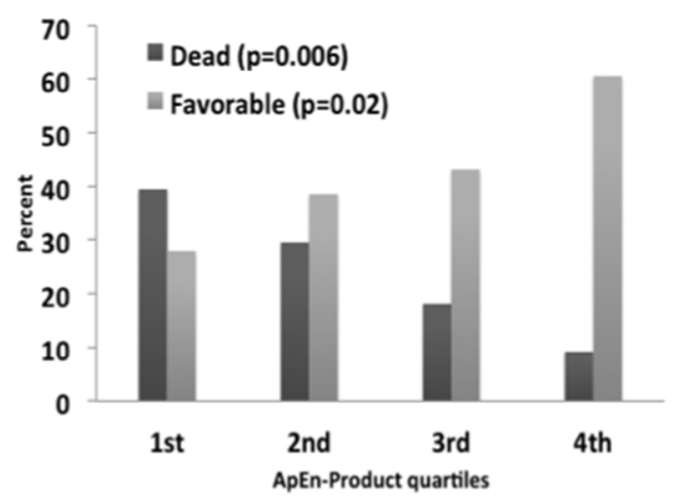

$254 \times 338 \mathrm{~mm}(72 \times 72 \mathrm{DPI})$ 
Figure 1. Illustrations represent the same original time series, considered as (A) single points, $m$ $=1$ or $(B)$ double points, $m+1=2$. For $m=1$, at points 4 and $5[u(4)$ and $u(5)]$, there is one other point $[u(9)$ and $u(6)$, respectively] that fall within a set standard deviation $( \pm r=20 \%)$ of $u(4)$ and $u(5)$. The probability this occurs is calculated for each point from 1 to $N$. For $m+1=2, u(4)$ and $u(5)$ are considered together. A search is made for two consecutive points that lie within the set $( \pm r=20 \%)$ standard deviation; none are seen in B. A mean probability is calculated from this for each pair of points in the time series. The approximate entropy is then the natural logarithmic difference between the two probabilities, in other words, what is the chance a similar runs (filter $r$ ) of length $m$, also remain similar when length of run is increased by 1 .

Figure 2. Calculated parameters for the first 6-hour monitoring period separated by Glasgow Outcome Scale groups. Intracranial pressure (ICP), cerebral perfusion pressure (CPP), pressure reactivity index (PRx), approximate entropy (ApEn) for ICP, MAP and HR. Results represent mean and $95 \%$ confidence intervals. Symbols express the difference between groups in one-way ANOVA followed by Bonferroni post hoc analysis with a confidence level of $P<0.05$. * Differed from Good Outcome.

Figure 3. Empirical regression plots between complexity and pressure reactivity index (PRx). (A) Approximate entropy of intracranial pressure (ApEn-ICP), (B) Approximate entropy of mean arterial pressure (ApEn-MAP), and (C) Approximate entropy of heart rate (ApEn-HR). Results are mean \pm SEM. 
Figure 4. Percentage of patients that were dead and those that had favourable outcome according to the approximate entropy product from $1^{\text {st }}$ to $4^{\text {th }}$ quartile in 6 -hour analysis. $p$ values represent results of chi-square test for inter-quartile differences. 